\title{
FLEBOLITOS EN LA REGIÓN MAXILOFACIAL: UN DESAFÍO PARA EL DIAGNÓSTICO POR IMÁGENES. UNA REVISIÓN
}

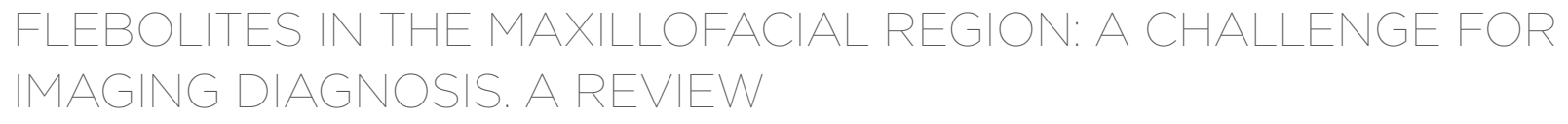

Jorge Luis Becerra-Heredia' * (i)

jorbecerra76@hotmail.com

\section{Gustavo Adolfo Fiori-Chíncaro ${ }^{2}$ gfiori@ilaeperu.com}

Ana María Agudelo-Botero 3,4 (i) ortoamariabotero@gmail.com

Artículo recibido: 17/10/2021

Arbitrado por pares

Artículo aceptado: 24/11/2021

Artículo publicado: 10/12/2021

* Autor corresponsal:

Jorge Luis Becerra Heredia

jorbecerra76@hotmail.com

\section{(c) (i)}

CLa autora, 2021. Publicado por la Universidad Científica del Sur (Lima, Perú)

\section{RESUMEN}

Las alteraciones o trastornos vasculares de los vasos sanguíneos o linfáticos presentan características propias, y la identificación de calcificaciones es un parámetro adecuado para realizar un diagnóstico certero. El objetivo del estudio fue describir las características radiográficas de los flebolitos y determinar si representan un desafío para el diagnóstico radiológico. Según la literatura revisada en las bases de datos de Medline (PubMed), SciELO, Google Escolar y algunas revistas especializadas del área, se determinó que los flebolitos tienen características radiográficas muy particulares, de imágenes concéntricas radiotransparentes y radiopacas que semejan a anillos. Sin embargo, no todas las presentaciones son similares, esto hace el diagnóstico engañoso por otras calcificaciones en esta región, como los sialolitos, cuyo aspecto suele ser parecido. A su vez, está la relación con anomalías vasculares y hemangiomas. Finalmente, la evidencia describe características muy propias de esta lesión, pero es necesario individualizar cada caso, por las diferencias que existe de un paciente a otro.

Palabras claves: flebolitos, malformaciones vasculares, hemangioma cavernoso, calcificación

\begin{abstract}
Vascular alterations or disorders of the blood and / or lymphatic vessels have their own characteristics, the identification of calcifications being a correct parameter to be able to make a proper diagnosis. The objective of the study was to describe the radiographic characteristics of the phleboliths, and whether they represent a challenge for diagnosis. according to the literature reviewed in the Medline (PubMed) database, Scielo, Google Scholar and some specialized journals. Phleboliths were found to have very particular radiographic characteristics, with concentric radiolucent and radiopaque images that resemble rings. However, not all the presentations are similar, this makes the diagnosis misleading with other calcifications in this region, such as sialoliths where their appearance is usually similar. Furthermore, there is the relationship with vascular anomalies and hemangiomas. finally, the evidence points to very specific characteristics of this lesion, and it is necessary to individualize each case, due to the differences that exist from one patient to another.
\end{abstract}

Keywords: phleboliths, vascular malformation, cavernous hemangioman, calcification

Citar como: Becerra-Heredia JL, FioriChíncaro GA, Agudelo-Botero AM. Flebolitos en la región maxilofacial, un desafío para el diagnóstico por imágenes: Una revisión. Rev Cient Odontol (Lima). 2021;9(4): e086.

DOI: $10.21142 / 2523-2754-0904-2021-086$

\footnotetext{
Facultad de Estomatología, Universidad Inca Garcilaso de la Vega. Lima, Perú.

2 Instituto Latinoamericano de Altos Estudios en Estomatología (ILAE). Lima, Perú.

Fundación Centro de Investigaciones y Estudios Odontológicos CIEO. Bogotá, Colombia.

4 Facultad de Estomatología, Universidad Militar Nueva Granada. Bogotá, Colombia.
} 


\section{INTRODUCCIÓN}

Los flebolitos son nódulos calcificados idiopáticos poco comunes en la región maxilofacial y se caracterizan por presentar calcificaciones concéntricas radiotransparentes de forma ovalada o circular. Se forman por la acumulación de minerales en un trombo; además, están relacionados con anomalías vasculares (AV), hemangiomas y malformaciones vasculares, o por traumatismos, $y$ se forman por estancamiento vascular $\left.{ }^{(1-9}\right)$.

Estas AV son alteraciones o trastornos vasculares caracterizados por un desarrollo anormal de los vasos sanguíneos o linfáticos, y la mayoría presentan características propias. La presencia de calcificaciones favorece al camino correcto para el diagnóstico de AV. Se pueden presentar tanto en lactantes como en acianos, pero con frecuencia entre la primera y la tercera década de vida. Aunque no hay predilección por el sexo, se le ha reconocido un alto porcentaje de relación con los músculos masticatorios $\left({ }^{3,5,10-12}\right)$.

Para las AV, en 1982, Mulliken y Glowacki plantearon una clasificación biológica basada en su comportamiento, presentación clínica y características patológicas predominantes en el endotelio, y las dividieron en dos grandes grupos: hemangiomas y malformaciones vasculares $(6,11,13-16)$.

Los flebolitos relacionados con AV fueron encontrados inicialmente por Constatt, en 1843, en la vena esplénica, y en la región maxilofacial por Kirmission, en 1905. Si bien son muy frecuentes en venas pélvicas, son raras en la región maxilofacial, y en ocasiones se pueden localizar en hemangiomas, pero es patognomónico de las malformaciones vasculares de bajo flujo $\left(^{6}\right)$.

Los hemangiomas son los tumores más comunes de la infancia y se presentan en el 4 al 10\% de los niños, como vasos recién desarrollados de células endoteliales hiperplásicas. Un alto porcentaje aparece en cabeza y cuello, y se diagnostica en el primer año de vida. Se caracterizan por un crecimiento rápido en los primeros meses, seguido por procesos de involución y un desarrollo lento acompañado de más cambios. Un flebolito puede estar asociado a un hemangioma residual de la infancia que podría manifestarse en la adultez $\left({ }^{6,7,11}\right)$.

Las malformaciones vasculares en la región maxilofacial representan el $40 \%$ de todos los casos. La tasa de incidencia es de 1 a 2 casos por cada 10000 nacidos vivos. Las malformaciones venosas son las más comunes de este grupo y afectan al $1 \%$ de la población. Se caracterizan por un desarrollo anormal de las venas con presencia de estancamientos $\left({ }^{15}\right)$. Esta condición va a favorecer la formación de flebolitos a nivel vascular, lo que es una característica imagenológica para este tipo de malformación $(4,6,7,17)$.

Los exámenes de imágenes cumplen roles importantes para definir el diagnóstico de AV y flebolitos, los datos clínicos del paciente en la historia clínica resultan de mucha ayuda $\left({ }^{3,10}\right)$ y los rayos $\mathrm{X}$ de rutina nos dan un diagnóstico inicial. Se presentan como zonas radiopacas, ovaladas concéntricas, cerca de piezas dentales y del ángulo mandibular. La tomografía computarizada (TC) facilita imágenes hipodensas y nítidas de estas calcificaciones, la resonancia magnética (RM) brinda imágenes hiperintensas bien definidas, y el eco Doppler pueden darnos imágenes de pequeños focos hiperecoicos de calcificaciones. Además de evaluar su ubicación, amplitud y límites con estructuras vecinas, todos estos exámenes de imágenes cumplen un rol muy importante para llegar a un consenso en el diagnóstico de estas anomalías y permiten delimitar el camino para su manejo adecuado $(3,4,18,19)$.

Con base en este contexto, el propósito de la investigación se centrará, mediante una revisión narrativa, en describir el diagnóstico de los casos reportados con flebolitos e identificar y conocer sus características imagenológicas en la región maxilofacial con los métodos de imágenes existentes, a fin de que el cirujano dentista tenga la información y el conocimiento básico y especializado de estas calcificaciones para dar un diagnóstico inicial oportuno. 


\section{MATERIALES Y MÉTODOS}

Se realizó una búsqueda bibliográfica en las principales de bases de datos de la literatura científica en ciencias de la salud: Medline a través de PubMed, Google Escolar y SciELO, utilizando las palabras clave "flebolitos", "malformación vascular", "hemangioma cavernoso" y "radiografía panorámica". Del mismo modo, se incluyeron artículos en inglés y español publicados hasta el 31de agosto de 2021.

\section{Etiología, aspectos epidemiológicos y demográficos}

Los flebolitos son lesiones poco comunes en la región maxilofacial. Se caracterizan por un alto grado de calcificación y se piensa que se forman por la calcificación de trombos intravenosos $\left({ }^{4}\right)$. Su estructura se asemeja a una masa calcificada de forma redondeada, que con frecuencia exhibe una disposición laminar. $\mathrm{Su}$ pico alto de crecimiento está dado por la influencia de los fibroblastos y su crecimiento progresa del interior hacia el exterior. Están asociados con lesiones vasculares como los hemangiomas y las malformaciones vasculares (3, 6-8, ${ }^{20}$ ). La disminución del flujo sanguíneo en las AV genera trombos y flebolitos. Su patogenia se transformó a partir de la teoría de Ribbert, quien demostró que los flebolitos aparecen en trombos y se relacionan con las paredes vasculares, donde se mineralizan y desarrollan $\left({ }^{4,6}\right)$.

$\mathrm{Su}$ localización o incidencia en cabeza y cuello representa de un 5 a un 20\%, con un promedio del 13,5\%; en hemangiomas cavernosos, de un 30 a un 50\%; y en malformaciones vasculares de tejidos blandos, un $20 \%$. Esta entidad no se asocia con factores hereditarios y lo relacionan más con la falta de apoyo vascular y el estancamiento venoso. Su crecimiento es lento y no presenta sintomatología, se relaciona con los músculos masticatorios y mejilla (27,6\%). Aunque son raros en glándulas salivales, se han reportado casos en forma de masas depresibles, edema y, en ocasiones, sintomáticos. Esta calcificación se puede presentar a cualquier edad, de preferencia en la primera y tercera década de vida $(55,2 \%), y$ no existe predilección por raza o sexo $(3,4,6$, $7,15,21)$.
Su incidencia es del $5 \%$ al $20 \%$ en la región de cabeza y cuello. Su relación con los hemangiomas es del 5\% del total de todos los casos de cirugía oral. La región de la mejilla se considera el sitio más común de flebolitos, seguido por las glándulas salivales parótidas, la lengua, los labios y la tiroides. Aunque los flebolitos son raros en glándulas salivales, se han reportado algunos casos. Los flebolitos que se originan en las glándulas salivales pueden causar masas depresibles y edemas que en ocasiones son sintomáticos. La mayoría de estas calcificaciones en la región maxilofacial se encuentran en formación de grupos; por el contrario, los casos raros son solitarios. Una particularidad de la presencia de trombos son los altos niveles de dímero $\mathrm{D}$ en sangre, que están presentes en un total del $42 \%$ de las malformaciones vasculares. En la actualidad no se han reportado casos de mutación maligna ni de recidivas de flebolitos; sin embargo, es importante hacer seguimiento radiográfico $(4,6,9)$.

\section{CARACTERÍSTICAS CLÍNICAS E HISTOLÓGICAS}

Los flebolitos son calcificaciones benignas, que comúnmente se presentan como múltiples lesiones cálcicas, rara vez son solitarios y se relacionan con malformaciones vasculares. En la región craneofacial se presentan en un 13,5\% y suelen visualizarse en diferentes regiones del paciente y, generalmente, se localizan a través de descubrimientos en exámenes de imágenes de rutina.

Clínicamente, estas calcificaciones pueden pasar inadvertidas durante mucho tiempo, sin presencia de signos y son evidentes en hallazgos radiográficos. Según la ubicación, se puede presentar en forma de masas blandas o nódulos fluctuantes, de color rojizo o de un color violáceo, algunos de consistencia dura, muestran un desarrollo pausado y limitado. En la mayoría de los casos no se observan síntomas, suelen alterar la imagen facial de los pacientes si la lesión incrementa su volumen $\left({ }^{7,22,23}\right)$.

Histológicamente, los flebolitos se observan como calcificaciones concéntricas con un patrón laminar con apariencia de cebolla dentro de un vaso, lo que da la 
apariencia de ojo de buey, y tiene una forma redonda de color marrón rojizo brillante. La lámina externa está formada por tejido conectivo fibroso y de superficie lisa en su interior, que además está compuesta por un tejido blando elástico de color marrón brillante. Este tejido está lleno de glóbulos rojos laminados que forman un trombo organizado y, en el centro de la lesión, hay un cuerpo pequeño parecido a un cálculo incrustado, donde por acciones repetidas va creciendo este tipo de calcificación $(3,9,20,24,25)$.

\section{DIAGNÓSTICO DIFERENCIAL}

La presencia de flebolitos en la región maxilofacial y en tejidos blandos puede ser confusa por la presencia de múltiples calcificaciones, osificaciones y cuerpos extraños que se debe tomar en cuenta en el diagnóstico diferencial. Las calcificaciones son las más frecuentes que se caracterizan por la acumulación de sales de calcio en tejidos y algunas glándulas. Se pueden depositar por mecanismos metastásicos, distróficos o de naturaleza desconocida. Entre las calcificaciones se incluyen entidades como los sialolitos, cálculos calcificados, radiopacos, de forma redondeada y que ocasionan obstrucciones en las glándulas salivales; se caracterizan por el desarrollo de cálculos en la arquitectura ductal glandular o parénquima, se presentan con hinchazón intermitente para estos casos la sialografía sería la herramienta ideal para diagnosticar esta entidad $(3,5,16$, 20, 24, 26-28).

Los nódulos linfáticos calcificados son otra alternativa, y los más afectados son los cervicales y los submandibulares. Se han encontrado también nódulos linfáticos parotídeos debido a procesos inflamatorios crónicos, que es relativamente raro y está asociado principalmente con abscesos necróticos y tuberculosis. La miositis osificante traumática es una entidad de tipo distrófica que puede involucrar a músculos maseteros, pterigoideo medial, esternocleidomastoideo y temporal. Tiene un patrón plumoso a lo largo de las fibras musculares y se dice que es una condición rara causada por traumatismos. También otra patología de confusión es la linfadenopatía, asociada con la tuberculosis, pero que forma un patrón en forma de coliflor en una cadena que la diferencia del resto $\left({ }^{20,29}\right)$.

\section{ASPECTOS IMAGENOLÓGICOS}

Imagenológicamente, los flebolitos se observan dependiendo del tamaño; los más pequeños se pueden apreciar como cuerpos radiopacos de forma redondeada $\mathrm{u}$ ovalada, que con frecuencia se localizan a nivel del ángulo mandibular, los de mayor tamaño como cuerpos calcificados de forma redonda u ovoide tienen múltiples laminados que se distribuyen al azar, y de forma circular en el interior con diferenciación de halo radiopaco en el contorno y radiolúcido en el medio, que se asemejan a un ojo de un buey $\left({ }^{5,24}\right)$. En ocasiones, tiene un núcleo radiopaco o radiolúcido, y la repetición de estas calcificaciones tiene una apariencia de anillos, su presentación es múltiple; sin embargo, se han reportado casos únicos. Se presenta con mayor frecuencia en las mejillas, seguido de la parótida y otros tejidos bucales ( ${ }^{3}$, $5,16,24,25,28,30)$.

La tomografía computarizada (TC), por su sensibilidad, es otra herramienta clave para el diagnóstico de tejidos duros y calcificaciones, como es el caso del flebolito y demás afecciones. En esta se observa como múltiples imágenes hiperdensas circulares $u$ ovalados con diámetros diferentes distribuidas en grupos al azar; en ocasiones pueden ser únicos $(6,7,28)$.

La resonancia magnética $(\mathrm{RM})$, más que para la detección de flebolitos, es una herramienta para la detección de tejidos blandos teniendo en cuenta zonas superficiales y profundas $\left({ }^{28}\right)$, y en este caso en especial tumores $y$ malformaciones vasculares que están relacionadas con los flebolitos. Su principal característica es la presencia de cavitaciones y vacíos de forma redondeada u ovalada, zonas hiperintensas sin vacíos de flujos que confirman la característica de los flebolitos. Finalmente, podemos mencionar que además de los exámenes auxiliares por imágenes una biopsia seguida de un estudio microscópico ayudaría al diagnóstico final $(28,31)$.

Como datos históricos adicionales se puede mencionar que los métodos diagnósticos han evolucionado con el tiempo, y muestra de ello se observa que los exámenes de imágenes y equipos de diagnóstico han superado estándares antes pensados, lo que brinda tecnología avanzada por el bien de la salud. Al ser así, la literatura 


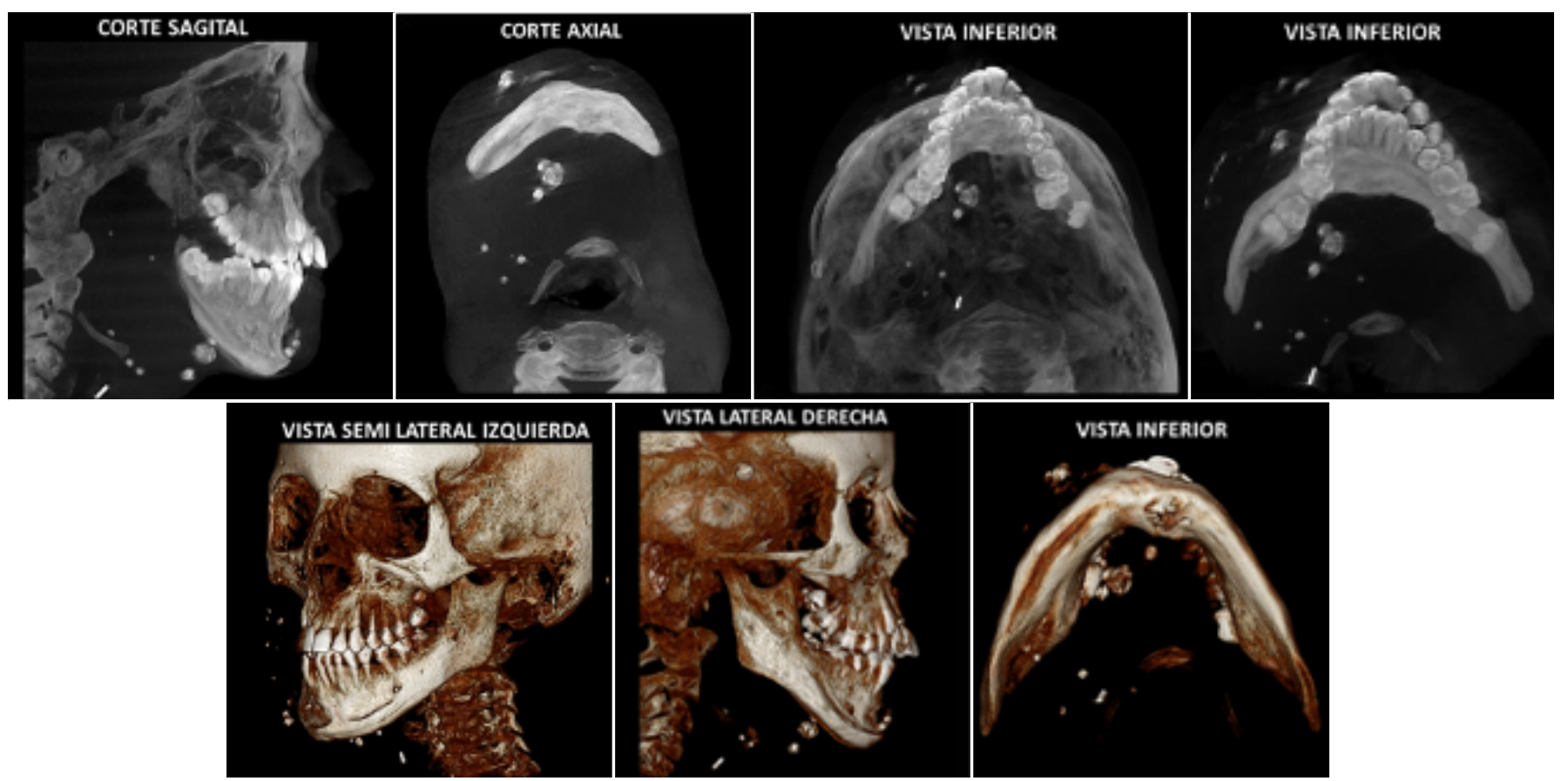

Figura I. Múltiples calcificaciones en región maxilofacial, flebolitos. Cortesía del Dr. Hugo Aguayo del Centro de Diagnóstico por Imágenes (CDI), Lima, Perú.

Tabla 1. Descripción de flebolitos reportados en la literatura científica en este nuevo milenio

\begin{tabular}{|c|c|c|c|c|c|c|c|c|c|}
\hline & Año & Edad & Sexo & Localización & Tamaño (mm) & Dolor & Edema & Imagen & Tratamiento \\
\hline Unal $\left({ }^{32}\right)$ & 2003 & 18 & M & Sublingual & $25 \times 15$ & - & + & TC RM & Cirugía \\
\hline Chuang $\left({ }^{33}\right)$ & 2005 & 65 & $M$ & Submandibular & & + & + & TC & Enucleación \\
\hline \multirow{2}{*}{ Altug $\left({ }^{34}\right)$} & & 22 & M & & & - & + & TC 2D & Ninguno \\
\hline & & 21 & M & SCM & & - & + & $\mathrm{TC}$ & Ninguno \\
\hline $\mathrm{Su}\left({ }^{35}\right)$ & 2009 & 23 & $\mathrm{~F}$ & Submandibular & & + & + & $2 \mathrm{DR}$ & Escleroterapia \\
\hline Mohan $\left({ }^{36}\right)$ & 2011 & 45 & $\mathrm{~F}$ & Mucosa & $2 \times 2$ & - & + & TC R & Ninguno \\
\hline Kato $\left({ }^{20}\right)$ & 2012 & 17 & $F$ & Masetero & $17 \times 14$ & - & + & $\mathrm{TC}$ & Cirugía \\
\hline Orhan $\left({ }^{37}\right)$ & 2012 & 20 & M & Múltiple & & & + & $\mathrm{TC}$ & Embolización \\
\hline Zengin $\left({ }^{28}\right)$ & 2013 & 21 & $\mathrm{~F}$ & Masetero & $2-10$ & - & + & RM & Ninguno \\
\hline Choi $\left.{ }^{(38}\right)$ & 2013 & 44 & M & Parótida & $60 \times 40$ & - & + & $\mathrm{TC}$ & Cirugía \\
\hline Chava $\left({ }^{39}\right)$ & 2013 & 28 & $M$ & Múltiples & $30 \times 20$ & - & + & $\mathrm{RM}$ & Ninguno \\
\hline $\operatorname{Lima}\left({ }^{3}\right)$ & 2015 & 56 & $\mathrm{~F}$ & Labio & 3 & - & + & & \\
\hline Ghosh $\left({ }^{23}\right)$ & 2015 & 37 & M & Múltiple & & - & + & RMAN & Ninguno \\
\hline Nagaraja $\left({ }^{41}\right)$ & 2016 & 49 & M & Bucal & $25 \times 15$ & - & - & $2 \mathrm{DR}$ & Cirugía \\
\hline Fernández $\left({ }^{6}\right)$ & 2016 & 9 & M & Parótida & & - & + & TC 2D & Escleroterapia \\
\hline Chen $\left({ }^{42}\right)$ & 2017 & 43 & $\mathrm{~F}$ & Parótida & 50 & - & + & $\mathrm{TC}$ & Cirugía \\
\hline Prakash $\left({ }^{43}\right)$ & 2017 & 30 & $M$ & Múltiple & $30 \times 40$ & + & + & 2D CD & Ninguno \\
\hline \multirow[t]{3}{*}{ Arroyo $\left({ }^{7}\right)$} & 2018 & 18 & M & R mentoniana & $40 \times 50$ & - & + & $\mathrm{TC}$ & Cirugía \\
\hline & & 17 & $\mathrm{~F}$ & Masetero & $35 \times 40$ & - & + & $\mathrm{TC}$ & Cirugía \\
\hline & & 17 & $\mathrm{~F}$ & R mentoniana & $3 \times 4$ & - & + & $\mathrm{TC}$ & Cirugía \\
\hline Sivrikaya $\left({ }^{2}\right)$ & 2019 & 26 & M & R bucal & 32 & - & + & 2D TC & Ninguno \\
\hline Shinichi $\left({ }^{4}\right)$ & 2020 & 66 & $\mathrm{~F}$ & A mandibular & & & + & 2D RM & Ninguno \\
\hline
\end{tabular}

Fuente: Literatura universal 
reporta múltiples casos en los últimos años, que resaltan estudios de imágenes más digitalizados y tecnificados que en años anteriores al milenio (tabla 1).

\section{CONCLUSIONES}

A pesar del índice bajo de calcificaciones en la región maxilofacial, los cirujanos dentistas deben conocer la existencia de estas entidades, y familiarizarse con las características radiográficas que presentan; del mismo modo, el diagnóstico diferencial, que causa controversias en su interpretación y diagnóstico. La búsqueda de información da a conocer que los flebolitos en la región maxilofacial son poco comunes, y se relacionan con hemangiomas y malformaciones vasculares, cabe destacar que estas entidades son identificadas como hallazgos radiográficos de rutina y que en diferentes circunstancias pasan por alto. Por lo tanto, es competencia del radiólogo maxilofacial reportar en sus informes estas calcificaciones, para que el clínico este familiarizado, y así informar a sus pacientes de la existencia de estas entidades y orientar como se debe actuar o remitir a la instancia correspondiente para su atención oportuna.

Contribución de autoría: Jorge Luis Becerra Heredia, Gustavo Adolfo Fiori-Chíncaro, y Ana María AgudeloBotero participaron en la elaboración y diseño del trabajo, así como la obtención y análisis de los datos, la revisión crítica del manuscrito y la aprobación de su versión final.

Potenciales conflictos de interés: Los autores declaran que no existe conflicto de interés en relación con la publicación de este artículo.

Fuente de financiamiento: Autofinanciado. 


\section{REFERENCIAS BIBLIOGRÁFICAS}

1. Gooi Z, Mydlarz WK, Tunkel DE, Eisele DW. Submandibular venous malformation phleboliths mimicking sialolithiasis in children.Laryngoscope.2014; 124(12): 2826-8.doi.org/10.1002/ lary. 24758

2. Sivrikaya EC, Cezairli B, Ayranci F, Omezli MM, Erzurumlu ZU. Buccal vascular malformation with multiple giant phleboliths: a rare case presentation and review of the literature. Oral Maxillofac Surg. 2019; 23(3): 375-80. doi.org/10.1007/ s10006-019-00767-0

3. Gouvêa Lima GdM, Moraes RM, Cavalcante ASR, Carvalho YR, Anbinder AL. An isolated phlebolith on the lip: an unusual case and review of the literature. Case Rep Pathol. 2015; 2015: 507840. doi.org/10.1155/2015/507840

4. Sato S, Takahashi M, Takahashi T. A case of multiple phleboliths on the medial side of the right mandible. Case Rep Dent. 2020; 2020: 6694402. doi.org/10.1155/2020/6694402

5. O'Riordan B. Phleboliths and salivary calculi. Br J Oral Surg. 1974; 12(2): 119-31. doi.org/10.1016/0007-117X(74)90120-6

6. Fernández RL, Rodríguez JT. ¿Flebolitos o sialolitos en la región maxilofacial? Revista Mexicana de Cirugía Bucal y Maxilofacial. 2016; 12(1): 28-32.

7. Chimalpopoca MTA, Rodríguez EP, Mancera NGI, Villanueva FAV. Flebolitos y revisión de tres casos. Revista Mexicana de Cirugía Bucal y Maxilofacial. 2018; 14(3): 126-34.

8. Mandel L, Perrino MA. Phleboliths and the vascular maxillofacial lesion. J Oral Maxillofac Surg. 2010; 68(8): 1973-6. doi.org/10.1016/j.joms.2010.04.002

9. Kamatani T, Saito T, Hamada Y, Kondo S, Shirota T, Shintani S. Intramuscular hemangioma with phleboliths of the tongue. Indian J Dent. 2014; 5(2): 100. doi: 10.4103/09.962X.135279

10. Brahmbhatt AN, Skalski KA, Bhatt AA. Vascular lesions of the head and neck: an update on classification and imaging review. Insights Imaging. 2020; 11(1): 19. doi.org/10.1186/s13244-0190818-3

11. Sadick M, Wohlgemuth WA, Huelse R, Lange B, Henzler T, Schoenberg SO, et al. Interdisciplinary Management of Head and Neck Vascular Anomalies: Clinical Presentation, Diagnostic Findings and Minimalinvasive Therapies. Eur J Radiol Open. 2017; 4: 63-8. doi.org/10.1016/j.ejro.2017.05.001

12. Redondo P (ed.). Clasificación de las anomalías vasculares (tumores y malformaciones): características clínicas e historia natural. Anales del Sistema Sanitario de Navarra; 2004: 27(1).

13. Park H, Kim JS, Park H, Kim JY, Huh S, Lee JM, et al. Venous malformations of the head and neck: a retrospective review of 82 cases. Arch Plast Surg. 2019; 46(1): 23. doi: 10.5999/ aps.2018.00458

14. Cortese A, Pantaleo G, D'Alessio G, Garzi A, Amato M. Hemangiomas of the maxillofacial area: case report, classification and treatment planning. Open Med (Wars). 2015; 10(1). doi. org/10.1515/med-2015-0066
15. Magaña FG, González JMM, Ugalde FAA, Ayala AA, Reyes AGI, Restrepo DV. Malformación venosa maxilar intraósea: reconstrucción microvascular (reporte de caso y revisión de la literatura). Revista Mexicana de Cirugía Bucal y Maxilofacial. 2018; 14(2): 77-83.

16. Scolozzi P, Laurent F, Lombardi T, Richter M. Intraoral venous malformation presenting with multiple phleboliths. Oral Surg Oral Med Oral Pathol Oral Radiol Endod. 2003; 96(2): 197200. doi.org/10.1016/S1079-2104(03)00098-2

17. Sierre S, Teplisky D, Lipsich J. Malformaciones vasculares: actualización en diagnóstico por imágenes y tratamiento. Archivos Argentinos de Pediatría. 2016; 114(2): 2-3.

18. Richter GT, Friedman AB. Hemangiomas and vascular malformations: current theory and management. Int J Pediatr. 2012; 2012: 645678. doi.org/10.1155/2012/645678

19. Müller-Wille R, Wildgruber M, Sadick M, Wohlgemuth WA (eds.). Vascular anomalies (part II): interventional therapy of peripheral vascular malformations. Rofo; 2018. doi:10.1055/s-0044-101266

20. Kato H, Ota Y, Sasaki M, Arai T, Sekido Y, Tsukinoki K. A phlebolith in the anterior portion of the masseter muscle. Tokai J Exp Clin Med. 2012; 37(1): 25-9.

21. Schwartz A, Salz N. Cavernous hemangioma associated with phleboliths in the masseter muscle. Acta radiol. 1955; 43(3): 2334. doi.org/10.3109/00016925509172765

22. Pérez-Macias Martín O, Peral Cagigal B, Madrigal Rubiales B, Blanco Sanfrutos S, Redondo González LM, Verrier Hernández A. Hemangioma cavernoso sublingual. Revista Española de Cirugía Oral y Maxilofacial. 2013;35(1): 44-6. doi.org/10.1016/j. maxilo.2011.10.004

23. Ghosh S, Singh K, Garg A, Kumar P, Gupta S. A rare case of low flow vascular malformation of head and neck region presenting with multiple phleboliths. J Clin Diagn Res. 2015; 9(6): ZJ01-2. doi: 7860/JCDR/2015/11806.6034

24. Calle Morocho JR, Montoya Gonzales DD, Calle Velezmoro EM. Calcificaciones de tejidos blandos: consideraciones diagnósticas. Revista Cubana de Estomatología. 2020; 57.

25. Parker Jr LA, Frommer HH. Phleboliths: Report of a case. Oral Surg Oral Med Oral Pathol. 1964; 18(4): 476-80. doi. org/10.1016/0030-4220(64)90394-9

26. Campos-Villanueva C, Téllez-Rodríguez J, López-Fernández R. Sialolitiasis submandibular en un paciente pediátrico. Reporte de caso. Acta Pediátrica de México. 2014; 35(5): 393-401.

27. Marchal F, Dulguerov P. Sialolithiasis Management: The State of the Art. Arch Otolaryngol Head Neck Surg. 2003; 129(9): 9516. doi: 10.1001/archotol.129.9.951

28. Zengin AZ, Celenk P, Sumer AP. Intramuscular hemangioma presenting with multiple phleboliths: a case report. Oral Surg Oral Med Oral Pathol Oral Radiol. 2013; 115(1): e32-e6. doi. org/10.1016/j.oooo.2012.02,032 
29. Janeiro-Barrera S, García-Sánchez A, Molina-Barraguer I, Ramos-Murguialday M, Morey-Mas MÁ, Iriarte-Ortabe JI. Miositis osificante traumática. Revista Española de Cirugía Oral y Maxilofacial. 2013; 35(3): 137-8. doi.org/10.1016/j. maxilo.2012.05.005

30. Garay I, Olate S. Consideraciones actuales en el estudio imagenológico de las calcificaciones de tejidos blandos en zona de ángulo mandibular. Int J Odontostomat. 2013; 7: 455-64. doi. org/10.4067/S0718-381X2013000300019

31. Fayad L, Hazirolan T, Bluemke D, Mitchell S. Vascular malformations in the extremities: emphasis on MR imaging features that guide treatment options. Skeletal Radiol. 2006; 35(3): 127-37. doi.org/10.1007/s00256-005-

32. Çankaya H, Ünal Ö, Ugras S, Yuca K, Kiris M. Hemangioma with phleboliths in the sublingual gland: as a cause of submental opacity. Tohoku J Exp Med. 2003;199(3):187-91. doi. org/10.1620/tjem.199.187

33. Chuang C-C, Lin H-C, Huang C-W. Submandibular cavernous hemangiomas with multiple phleboliths masquerading as sialolithiasis. J Chin Med Assoc. 2005; 68(9): 441-3. doi. org/10.1016/S1726-4901(09)70162-5

34. Altuğ HA, Büyüksoy V, Okçu KM, Doğan N. Hemangiomas of the head and neck with phleboliths: clinical features, diagnostic imaging, and treatment of 3 cases. Oral Surg Oral Med Oral Pathol Oral Radiol. 2007; 103(3): e60-e4. doi.org/10.1016/j. tripleo.2006.09.006

35. Su Yx, Liao Gq, Wang L, Liang Yj, Chu M, Zheng Gs. Sialoliths or phleboliths? Laryngoscope. 2009; 119(7): 1344-7. doi. org/10.1002/lary.20514
36. Mohan RPS, Dhillon M, Gill N. Intraoral venous malformation with phleboliths. The Saudi Dent J. 2011; 23(3): 161-3. doi. org/10.1016/j.sdentj.2011.02.003

37. Orhan K, Icen M, Aksoy S, Avsever H, Akcicek G. Large arteriovenous malformation of the oromaxillofacial region with multiple phleboliths. Oral Surg Oral Med Oral Pathol Oral Radiol. 2012; 114(4): e147-e58. doi.org/10.1016/j. oooo.2012.05.007

38. Choi HJ, Lee JC, Kim JH, Lee YM, Lee HJ. Cavernous hemangioma with large phlebolith of the parotid gland. J Craniofac Surg. 2013; 24(6): e621-e3. doi: 10.1097/ SCS.0b013e3182a2d87b

39. Chava VR, Shankar AN, Vemanna NS, Cholleti SK. Multiple venous malformations with phleboliths: radiologicalpathological correlation. J Clin Imaging Sci. 2013; 3(Suppl 1): 13. doi: $10.4103 / 2156-7514.124058$

40. Aynali G, Ünal F, Yariktaş̧ M. Submandibular hemangioma with multiple phleboliths mimicking sialolithiasis: the first pediatric case. Kulak Burun Bogaz Ihtis Derg. 2014; 24(3): 168-71. doi: 10.5606/kbbihtisas.2014.24392

41. Nagaraja A, Kumar NG, Kumar BJ, Naik RM, Sangineedi YJ. A solitary phlebolith in the buccal mucosa: report of a rare entity and clinicopathologic correlation. J Contemp Dent Pract. 2016; 17(8): 706-10. doi.10.5005/jp-journals-10024-1916

42. Bo C. Cavernous hemangioma with multiple phleboliths of the parotid gland in adult masquerading assialolithiasis. Int J Clin Exp Med. 2017; 10(7): 11097-100.

43. Prakash S, Naik V, Dhanave J. Multiple Low Flow Vascular Malformation with Phleboliths-A Case Report. Journal of Young Pharmacists. 2017; 9(3): 446-50. doi: 10.5530/jyp.2017.9.87 